\title{
Economic costs of chemotherapy-induced febrile neutropenia among patients with non-Hodgkin's lymphoma in European and Australian clinical practice
}

Derek Weycker ${ }^{1 *}$, Aurelie Danel${ }^{2}$, Anne Marciniak ${ }^{3}$, Kate Bendall ${ }^{4}$, Michael Lipsitz ${ }^{1}$ and Ruth Pettengell ${ }^{5}$

\begin{abstract}
Background: Economic implications of chemotherapy-induced febrile neutropenia (FN) in European and Australian clinical practice are largely unknown.

Methods: Data were obtained from a European (97\%) and Australian (3\%) observational study of patients with non-Hodgkin's lymphoma (NHL) receiving CHOP ( \pm rituximab) chemotherapy. For each patient, each cycle of chemotherapy within the course, and each occurrence of FN within cycles, was identified. Patients developing FN in a given cycle ("FN patients"), starting with the first, were matched to those who did not develop FN in that cycle ("comparison patients"), irrespective of subsequent FN events. FN-related healthcare costs (£2010) were tallied for the initial FN event as well as follow-on care and FN events in subsequent cycles.
\end{abstract}

Results: Mean total cost was $£ 5776(95 \% \mathrm{Cl} £ 44928-£ 6713)$ higher for FN patients ( $n=295)$ versus comparison patients, comprising $£ 4051$ ( $£ 3633-£ 4485$ ) for the initial event and a difference of $£ 1725$ ( $£ 978-£ 2498$ ) in subsequent cycles. Among FN patients requiring inpatient care (76\% of all FN patients), mean total cost was higher by $£ 7259$ ( $£ 6327-£ 8205$ ), comprising $£ 5281$ ( $£ 4810-£ 5774)$ for the initial hospitalization and a difference of $£ 1978$ (£1262-£2801) in subsequent cycles.

Conclusions: Cost of chemotherapy-induced FN among NHL patients in European and Australian clinical practice is substantial; a sizable percentage is attributable to follow-on care and subsequent FN events.

Keywords: Febrile neutropenia, Costs and cost analysis, Non-Hodgkin's lymphoma

\section{Background}

Neutropenia is a common side effect of myelosuppressive chemotherapy. Neutropenia both increases the risk of infection and diminishes patients' ability to fight infection. Since fever is a cardinal sign of infection, when neutropenic patients develop fever (i.e., febrile neutropenia $[\mathrm{FN}])$, the high likelihood of infection and serious consequences usually necessitates hospitalization for urgent evaluation, ongoing monitoring, and administration of intravenous antibiotics. FN, as well as severe or prolonged neutropenia, can lead to dose-delays, dose-reductions, and/or chemotherapy discontinuations, interfering with

\footnotetext{
* Correspondence: dweycker@pai2.com

'Policy Analysis Inc. (PAI), 4 Davis Court, Brookline, MA 02445, USA Full list of author information is available at the end of the article
}

the delivery of optimal treatment and possibly adversely affecting patient outcomes [1-5].

The economic cost of chemotherapy-induced FN is substantial [6-8]. In one study, the mean cost of FN-related hospitalizations in the US was reported to be $\$ 8100$ for patients with solid tumors, $\$ 11,600$ for patients with nonHodgkin's lymphoma (NHL), and $\$ 28,000$ for patients with leukemia [7]. It is likely, however, that this study--as well as other studies utilizing a cross-sectional design and hospital records--underestimated the total economic burden of FN, since the cost of follow-on care (e.g., postdischarge outpatient encounters) and subsequent FN events that may be related to the (initial) FN event were not considered. In a more recent US study employing a matched cohort design and considering all such costs, the 
economic burden of the initial FN event was estimated to represent only about $60 \%$ of the total cost of FNrelated care, suggesting that prior studies may have underestimated the mean cost of FN-related events by as much as $40 \%$ [9].

Relatively little is known about the economic burden of chemotherapy-induced FN in European and Australian clinical practice, however, and no studies conducted outside the US have considered the burden of care for the initial FN event as well as the downstream consequences of the initial event. The current analysis, therefore, was undertaken to evaluate the full economic impact of FN among NHL patients receiving chemotherapy with cyclophosphamide, doxorubicin, vincristine, and prednisolone every 14 or 21 days (i.e., CHOP-14 or CHOP-21) in geographically diverse centers across Europe and in Australia.

\section{Materials and methods}

\section{Study setting and source population}

Data for this study were obtained from a large combined retrospective/prospective observational evaluation of supportive care among NHL patients receiving CHOP14 or CHOP-21 chemotherapy in geographically diverse centers primarily across Western and Southern Europe (97\% of patients) and in Australia (3\% of patients). The design of this study, termed the "IMPACT NHL Study" (ClinicalTrials.gov: NCT00903812), is described in detail elsewhere in the literature [10-13]. In countries where it was required, ethical approval of the IMPACT NHL Study was obtained and patients provided written informed consent.

Briefly, approximately 1800 adult patients with NHL who were planned to receive at least three cycles of CHOP-14 or CHOP-21 chemotherapy--with or without rituximab--were recruited (about 600 retrospectively, 1200 prospectively) from 128 geographically diverse study centers. Study patients may have received a prior course of chemotherapy ( $9 \%$ of study population). Patients enrolled retrospectively included those who completed all cycles of chemotherapy--regardless of actual number of cycles or outcomes thereof--after January 1, 2005 and prior to site participation in the study. Patients enrolled prospectively included all patients for whom CHOP-14 or CHOP-21 chemotherapy was planned for administration between January 2006 and December 2008; no additional interventions were required as a result of participation in the study. All patients enrolled in the IMPACT NHL Study $(\mathrm{n}=1,864)$ who met protocoldefined eligibility criteria and received at least one cycle of chemotherapy $(n=1,829)$ were included in the source population for the current analysis.

For each patient, baseline data were collected on patient demographics (e.g., age, sex, race, height, weight), medical history (e.g., comorbid conditions), Eastern Cooperative Oncology Group Performance Status (ECOG PS), cancer characteristics (e.g., bulky disease, bone marrow involvement), International Prognostic Index (IPI) score (non-follicular patients only)/Follicular Lymphoma International Prognostic Index (FLIPI) score (follicular patients only), prior antineoplastic therapy, planned chemotherapy (i.e., CHOP-14 or CHOP-21, with or without rituximab ["-R"], doses, number of cycles), and predicted risk of FN (i.e., $<20 \%$ vs $\geq 20 \%$ ). Cycle-specific data collected during the chemotherapy course included actual chemotherapy regimen (i.e., drug, dose, date of administration), use of selected drugs/services (e.g., granulocyte colony-stimulating factor [G-CSF] for prophylaxis [primary or secondary] or treatment, erythropoiesisstimulating agent [ESA], transfusions, anti-infective agents), performance status, and occurrence of FN--as well as selected other adverse outcomes--and associated setting of care (i.e., hospital, outpatient setting, home). Hospitalizations for reasons other than FN were also captured.

\section{Study population}

The study population, consisting of patients who experienced FN and matched comparison patients, was selected from the source population as follows. For each patient in the source population, all chemotherapy cycles in which FN occurred were identified. FN was defined as a single oral temperature of $\geq 38.3^{\circ} \mathrm{C}$ or a temperature of $\geq 38.0^{\circ} \mathrm{C}$ for $\geq 1$ hour, and a neutrophil count of $<0.5 \mathrm{x}$ $10^{9} / \mathrm{L}$ or a neutrophil count of $<1.0 \times 10^{9} / \mathrm{L}$ that is predicted to fall below $0.5 \times 10^{9} / \mathrm{L}$. Patients with FN in more than one cycle were classified according to the cycle number in which FN first occurred.

Patients in the source population who developed FN during their first cycle of chemotherapy ("FN patients") were matched on selected covariates--hypothesized to be possibly associated with FN and healthcare costs--to patients who did not develop FN during their first cycle of chemotherapy ("comparison patients"), whether or not they developed FN in any subsequent cycles. Matched cycle-one FN patients and comparison patients were then removed from their respective pools. From remaining patients in the source population (i.e., those not previously matched), those who first developed FN in their second cycle of chemotherapy were matched to those who did not develop FN in that cycle. Matched cycle-two FN patients and comparison patients were then removed from their respective pools. FN patients and comparison patients were similarly matched in the third and all subsequent cycles. The cycle in which patients were matched was designated the "index cycle".

Matching was implemented--sequentially, on a cyclespecific basis--for each FN patient by identifying all candidate comparison patients matching that FN patient in 
terms of age ( \pm 5 years), tumor stage, and chemotherapy regimen. The candidate with the closest propensity score ("nearest neighbor") to the FN patient was selected as the matched comparison patient. Matching was conducted for FN patients randomly, and without replacement of comparison patients (i.e., comparison patients were matched to one FN patient only). Propensity scores were estimated using multivariate logistic regression; independent variables included all demographic and disease-specific characteristics (e.g., age, sex, IPI/FLIPI score, bone marrow involvement), predicted risk of FN, presence of (current/continuing) comorbidities, chemotherapy regimen, supportive care, ECOG PS, absolute neutrophil count (ANC), and presence of anemia symptoms (e.g., fatigue/tiredness, pallor/pale skin, headache, dyspnea). All of the above-listed variables--with the exception of ANC, presence of anemia symptoms, and supportive care--were characterised using data collected at baseline in the IMPACT NHL Study; the exceptions were characterised using data collected during the preindex cycles and index cycle, as appropriate.

An alternative, more restrictive matching procedure-including age ( \pm 5 years), country of residence, tumor stage, bone marrow involvement, IPI/FLIPI score, and chemotherapy regimen when identifying candidate comparison patients--was first employed, but was ultimately deemed by study investigators to be inadequate due to small sample size $(n=118)$. The characteristics of FN patients and comparison patients matched using this more restrictive procedure is described in Additional file 1: Table S1 of the online supplement.

\section{Study measures}

FN-related healthcare utilization was tallied for each FN patient and matched comparison patient from the cycle in which FN first occurred (for the former) through the last cycle of chemotherapy. Healthcare utilisation was examined in terms of: the number of FN events requiring inpatient care, outpatient care, home care, and other/unknown care, respectively; the total number of hospital days for all FN-related admissions that occurred from the index cycle through the end of the last chemotherapy cycle; and use--and reason for use--of G-CSF (pegfilgrastim, filgrastim, and other G-CSF [presumably lenograstim]) and IV antimicrobial agents by setting of care. Use of G-CSF and antimicrobial agents as prophylaxis was considered in post-index cycles only, while use of these agents as treatment was considered beginning with the index cycle.

FN-related healthcare costs were calculated for FN patients and comparison patients by combining estimates of FN-related healthcare utilization--as described above--with unit-cost data from the United Kingdom (UK). UK-specific unit costs were estimated from the perspective of the National Health Service using data from readily-available secondary sources and published literature, where available, as well as expert opinion, where needed. Principal sources of cost data were the 2009-2010 National Health Service (NHS) Schedule of Reference Costs (hospital costs), the 2010 Unit Costs of Health and Social Care Report from the Personal Social Services Research Unit (outpatient, home care, and other setting costs), and the British National Formulary (BNF), 60th Edition (pharmacotherapy costs) [14]. Unit costs are set forth in Table 1; a full description of methods employed to estimate unit costs is provided in Additional file 1: Table S2 of the online supplement.

\section{Statistical analyses}

The adequacy of the matching procedure was evaluated based on the similarity of matched FN and comparison patients in terms of their baseline characteristics. Categorical variables were compared using the McNemar or Bowker test, and continuous variables were compared using the paired-samples $t$-test. The similarity of matched patients and unmatched patients--in terms of their characteristics--also was examined.

FN-related healthcare utilization and costs from the index cycle through the end of the last cycle of chemotherapy were examined on a cumulative basis for each patient in the FN and comparison groups. Mean values were estimated for the two groups, as well as for differences between groups, on an overall basis during the index cycle and during post-index cycles. Hospital days for FN patients and comparison patients with missing data on admission/discharge dates were imputed based on corresponding subgroup-specific mean values. FNrelated healthcare costs also were evaluated among subgroups of patients defined on the basis of key characteristics, including index cycle, chemotherapy regimen, and predicted FN risk. Ninety-five percent confidence intervals (95\% CIs) were generated using techniques of nonparametric bootstrapping.

The appropriateness of combining FN-related healthcare utilization data from different countries with unit cost information from the UK was evaluated based on a comparison of country-specific mean numbers of patients developing FN by setting of care, and for patients requiring inpatient care, country-specific mean numbers of hospital days. These analyses were based on data from FN patients and comparison patients who were matched on the basis of the criteria set forth above (i.e., age, tumor stage, chemotherapy regimen, and propensity score) as well as country of residence. Results were summarized using means and 95\% CIs; formal statistical tests for heterogeneity between patients in different countries were not undertaken due to small country-specific sample sizes. 


\section{Results}

Patient characteristics

Among the 1829 NHL patients in the source population, 331 (18\%) experienced one or more FN events--a total of $479 \mathrm{FN}$ events--during their course of chemotherapy; the number of events, by cycle, occurred as follows: 1st cycle, 128 (27\%); 2nd cycle, 64 (13\%); 3rd cycle, 67 (14\%); 4th cycle, 68 (14\%); and 5th or later cycle, 152 (32\%). The majority of FN events (77\%) required inpatient care.

A total of $295 \mathrm{FN}$ patients were successfully matched (1:1)--on age ( \pm 5 years), tumor stage, and chemotherapy regimen, as well as closest propensity score--to comparison patients (Table 2). (Selected covariates are presented in Table 2; a full listing of covariates is provided in Additional file 1: Table S3 of the online supplement.) Mean $( \pm$ SD) age was $63( \pm 12)$ years, $69 \%$ had stage III or IV disease, and $91 \%$ received CHOP-14-R (28\%) or CHOP-21-R (63\%). No covariate differed significantly between FN patients and comparison patients. Most patients were matched in cycle $1(43 \%)$ or cycle $2(14 \%)$, and the mean number of cycles from the index cycle through the end of chemotherapy was $4.5( \pm 2.1)$ for FN patients and $4.6( \pm 2.1)$ for comparison patients. Ninetyseven percent of the study population received chemotherapy at a site in Western or Southern Europe; the remaining 3\% received care in Australia.

Matched and unmatched patients were similar in terms of many of their baseline characteristics, but matched patients were older (by about 4 years, on average) and a higher percentage of them had advanced disease $(58 \%$ vs $45 \%$ ) and a predicted risk of $\mathrm{FN} \geq 20 \%$ (73\% vs $61 \%$ ). These results were not unexpected, since age and presence of advanced disease are risk factors for FN, and comparison patients were matched to FN patients based on these factors. (Data on all baseline characteristics for matched and unmatched patients are available in Additional file 1: Table S4 of the online supplement.)

\section{FN-related healthcare utilization}

FN patients averaged 1.44 (95\%CI 1.34-1.56) FN events from their index cycle through the end of their course of chemotherapy--0.44 (0.34-0.56) during post-index cycles-and comparison patients averaged 0.15 (0.10-0.21) such events over the same period, corresponding to a difference of 1.29 FN events (1.17-1.43) (Table 3). Among FN patients, $76 \%$ of index FN events and $79 \%$ of post-index FN events required inpatient care; for comparison patients, the corresponding percentage (during post-index cycles) was $84 \%$. Among FN patients requiring inpatient care, the average number of FN events (irrespective of setting of care) was 1.10 (1.00-1.21) versus 0.13 (0.08-0.18) for matched comparison patients, a difference of 0.98 (0.85-1.11). Mean number of FN-related hospital days was 6.21 (5.28-7.17) for FN patients versus 0.63 (0.30-1.02) for comparison patients, a difference of 5.62 (4.64-6.62).

Levels of FN-related healthcare utilization among FN patients and comparison patients (matched on the

Table 1 UK-specific unit costs of FN-related care

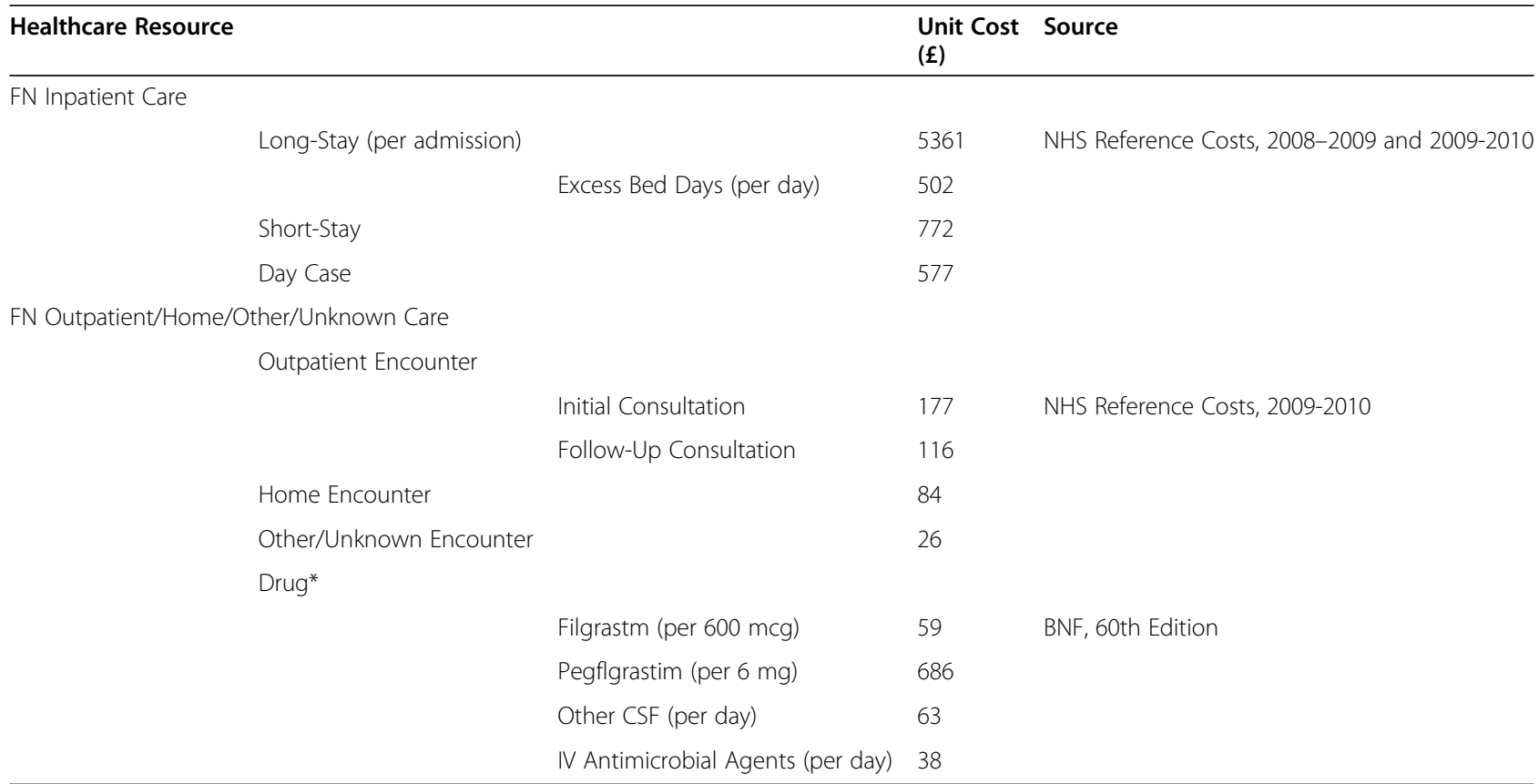


Table 2 Characteristics of FN patients and comparison patients matched on basis of age ( \pm 5 years), tumor stage, chemotherapy regimen, and propensity score

\begin{tabular}{|c|c|c|c|c|c|}
\hline & & & $\begin{array}{l}\text { FN Patients } \\
(\mathrm{n}=295)\end{array}$ & $\begin{array}{l}\text { Comparison } \\
\text { Patients } \\
(\mathrm{n}=295)\end{array}$ & P-value* \\
\hline \multicolumn{6}{|l|}{ Demographics } \\
\hline \multicolumn{3}{|l|}{ Age, mean $\pm S D, y$} & $\begin{array}{l}63.3 \pm \\
12.4\end{array}$ & $\begin{array}{l}63.0 \pm \\
12.1\end{array}$ & 0.108 \\
\hline \multicolumn{3}{|l|}{ Male, \% } & 49.0 & 50.7 & 0.674 \\
\hline \multicolumn{6}{|c|}{ Country of Residence } \\
\hline & \multicolumn{2}{|l|}{ Australia } & 2.7 & 2.7 & 0.817 \\
\hline & \multicolumn{2}{|l|}{ Austria } & 5.8 & 9.2 & \\
\hline & \multicolumn{2}{|l|}{ Belgium } & 11.6 & 11.9 & \\
\hline & \multicolumn{2}{|l|}{ France } & 24.5 & 18.4 & \\
\hline & \multicolumn{2}{|l|}{ Germany } & 10.2 & 12.2 & \\
\hline & \multicolumn{2}{|l|}{ Greece } & 2.7 & 1.7 & \\
\hline & \multicolumn{2}{|l|}{ Italy } & 5.1 & 7.8 & \\
\hline & \multicolumn{2}{|l|}{ Netherlands } & 8.8 & 8.5 & \\
\hline & \multicolumn{2}{|l|}{ Nordics } & 7.1 & 6.5 & \\
\hline & \multicolumn{2}{|l|}{ Portugal } & 1.4 & 1.7 & \\
\hline & \multicolumn{2}{|l|}{ Spain } & 12.6 & 12.6 & \\
\hline & \multicolumn{2}{|l|}{ Switzerland } & 2.7 & 2.4 & \\
\hline & \multicolumn{2}{|l|}{ UK and Ireland } & 4.8 & 4.4 & \\
\hline \multicolumn{6}{|l|}{$\mathrm{NHL}$} \\
\hline \multicolumn{6}{|l|}{ Tumor Stage } \\
\hline \multicolumn{3}{|l|}{ । } & 14.6 & 14.6 & - \\
\hline \multicolumn{3}{|l|}{$\|$} & 16.7 & 16.7 & \\
\hline \multicolumn{3}{|l|}{ III } & 19.4 & 19.4 & \\
\hline \multicolumn{3}{|l|}{ IV } & 49.3 & 49.3 & \\
\hline \multicolumn{6}{|l|}{ IPI/FLIPI Score } \\
\hline & \multicolumn{5}{|l|}{ IPI Score } \\
\hline & & Low & 16.4 & 20.8 & \multirow[t]{4}{*}{0.368} \\
\hline & & Intermediate & 50.4 & 50.6 & \\
\hline & & High & 19.6 & 17.3 & \\
\hline & & Missing & 13.6 & 11.4 & \\
\hline & FLIPI score & & & & \\
\hline & & Low Risk & 11.4 & 15.4 & 0.920 \\
\hline & & Intermediate Risk & 36.4 & 23.1 & \\
\hline & & Poor Risk & 43.2 & 51.3 & \\
\hline & & Missing & 9.1 & 10.3 & \\
\hline & Bone Marrow Involvement & & 29.6 & 24.8 & 0.252 \\
\hline Clinical & & & & & \\
\hline Risk of FN, \% & & & & & \\
\hline & $<20 \%$ & & 27.0 & 27.0 & 0.564 \\
\hline & $\geq 20 \%$ & & 73.0 & 73.0 & \\
\hline
\end{tabular}


Table 2 Characteristics of FN patients and comparison patients matched on basis of age ( \pm 5 years), tumor stage, chemotherapy regimen, and propensity score (Continued)

\begin{tabular}{|c|c|c|c|c|c|c|}
\hline & Cardiovascular Disease & & & 23.5 & 25.2 & 0.615 \\
\hline & Respiratory Disease & & & 7.8 & 4.8 & 0.128 \\
\hline & Gastrointestinal Disease & & & 6.1 & 5.1 & 0.564 \\
\hline & Renal Disease & & & 3.1 & 3.4 & 0.796 \\
\hline & Hepatic/Biliary Disease & & & 2.7 & 5.8 & 0.061 \\
\hline & Haematologic/Lymphatic & & & 5.1 & 3.1 & 0.221 \\
\hline & Immunologic & & & 5.4 & 3.7 & 0.317 \\
\hline & Other & & & 49.0 & 46.3 & 0.505 \\
\hline \multicolumn{7}{|l|}{ ECOG PS, \% } \\
\hline & 0 & & & 47.6 & 52.4 & 0.182 \\
\hline & 1 & & & 31.0 & 31.6 & \\
\hline & 2 & & & 10.2 & 7.5 & \\
\hline & 3 & & & 5.1 & 3.7 & \\
\hline & 4 & & & 1.0 & 0.7 & \\
\hline & Missing & & & 5.1 & 4.1 & \\
\hline \multicolumn{7}{|l|}{ Hematology } \\
\hline & ANC (X10^9L), mean \pm SD & & & $5.4 \pm 4.5$ & $5.7 \pm 3.4$ & 0.794 \\
\hline & Presence of Anemia Symptoms, \% & & & 56.8 & 56.1 & 0.856 \\
\hline \multicolumn{7}{|l|}{ Treatment } \\
\hline \multicolumn{7}{|c|}{ Chemotherapy Regimen, \% } \\
\hline & CHOP-14 & & & 3.40 & 3.40 & - \\
\hline & CHOP-14-R & & & 27.89 & 27.89 & \\
\hline & $\mathrm{CHOP}-21$ & & & 5.44 & 5.44 & \\
\hline & CHOP-21-R & & & 63.27 & 63.27 & \\
\hline \multicolumn{7}{|l|}{ Supportive Care, \% } \\
\hline & Index Cycle & & & & & \\
\hline & & G-CSF Prophylaxis & & 51.7 & 56.5 & 0.162 \\
\hline & & Anti-Infective FN Prophylaxis & & 13.3 & 11.6 & 0.529 \\
\hline & Pre-Index Cycle & & & & & \\
\hline & & G-CSF & & & & \\
\hline & & & Prophylaxis & 37.4 & 41.8 & 0.074 \\
\hline & & & Treatment & 9.5 & 6.5 & 0.128 \\
\hline & & Anti-Infective & & & & \\
\hline & & & FN Prophylaxis & 7.8 & 5.8 & 0.303 \\
\hline & & & FN Treatment & 1.7 & 0.3 & 0.102 \\
\hline & & Transfusion & & 9.5 & 6.8 & 0.144 \\
\hline \multicolumn{7}{|l|}{ Index Cycle Number* } \\
\hline 1 & & & & 42.9 & 42.9 & - \\
\hline 2 & & & & 13.6 & 13.6 & \\
\hline 3 & & & & 11.2 & 11.2 & \\
\hline 4 & & & & 12.2 & 12.2 & \\
\hline 5 & & & & 9.9 & 9.9 & \\
\hline 6 & & & & 8.2 & 8.2 & \\
\hline
\end{tabular}


Table 2 Characteristics of FN patients and comparison patients matched on basis of age ( \pm 5 years), tumor stage, chemotherapy regimen, and propensity score (Continued)

\begin{tabular}{|c|c|c|c|}
\hline 7 & 1.7 & 1.7 & \\
\hline 8 & 0.3 & 0.3 & \\
\hline \multicolumn{4}{|c|}{ Total Number of Cycles (incl. index cycle thru end of course) } \\
\hline 1 & 10.6 & 11.2 & 0.812 \\
\hline 2 & 11.9 & 9.5 & \\
\hline 3 & 13.3 & 11.6 & \\
\hline 4 & 12.3 & 15.3 & \\
\hline 5 & 12.6 & 11.6 & \\
\hline 6 & 24.2 & 24.8 & \\
\hline 7 & 4.1 & 4.1 & \\
\hline 8 & 10.9 & 11.9 & \\
\hline
\end{tabular}

*P-values were not calculated for variables used in the matching process and for which there are no differences between groups (i.e., there was an exact match).

basis of the criteria set forth above plus country of residence $[n=236]$ ) and corresponding differences between these groups, were largely comparable across countries (data available in Additional file 1: Table S5 of the online supplement). Among country-specific subgroups including $\geq 15$ patients, mean number of FN events among FN patients ranged from 1.41-1.63, and mean number of events requiring inpatient care, 0.791.50; the mean number of hospital days among FN patients ranged from 3.8-5.2.

\section{FN-related healthcare costs}

Mean overall FN-related healthcare cost among FN patients was $£ 8066$ (95\%CI $£ 7277-£ 8882)--£ 4051$ (£3633$£ 4485)$ during the index cycle and $£ 4015$ (£3374- £4724) during post-index cycles (Table 4). Mean cost of FN care among comparison patients (for whom all costs were incurred during post-index cycles, by design) was $£ 2290$ (£1923-£2655). Overall mean cost thus was $£ 5776$ (£4928-£6713) higher for FN patients than comparison patients, with $71 \%$ of the difference attributable to care in the index cycle $(£ 4051[£ 3633-£ 4485])$ and $29 \%$ attributable to care in post-index cycles ( $£ 1725$ [ $£ 978-£ 2498]$ ).

Stratified by the setting of care for the index FN event, the difference in mean overall cost was greatest for those who received inpatient care for FN during the index cycle versus comparison patients who were matched to them-- $£ 9688$ ( $£ 8709-£ 10,653)$ versus $£ 2430$ (£1984$£ 2931$ ), a difference of $£ 7259$ ( $£ 6327-£ 8205$ ). Corresponding results for those patients whose index FN event was treated in other settings of care were: outpatient, $£ 3761$ ( $£ 1908-£ 6007)$ versus $£ 1777$ (£1116$£ 2565)$; and home care, £3291 (£2445-£4339) versus $£ 1826$ (£993-£2919).
Differences in mean FN-related healthcare costs between FN patients and comparison patients were comparable within subgroups defined on the basis of key characteristics, including index cycle, chemotherapy regimen, and predicted FN risk. By index cycle, differences were: cycle 1, £6024 (£4588-£7429); cycle 2 , $£ 4913$ (£3123-£6875); cycle 3, £6029 (£3409-£9242); cycle 4, $£ 6804$ ( $£ 4519-£ 9543$ ); and cycle $\geq 5$, £5121 (£4084$£ 6220)$. For patients receiving CHOP-14-R and CHOP21-R, differences were $£ 5667$ ( $£ 4002-£ 7261)$ and $£ 6045$ (£4855-£7173), respectively. For matched FN and comparison patients with a predicted FN risk $\geq 20 \%$, difference was $£ 5603$ ( $£ 4603-£ 6605$ ), and for those with a predicted FN risk $<20 \%$, £6006 (£4557-£7783).

\section{Discussion}

Using a matched-cohort design, healthcare utilisation data for 1829 NHL patients receiving CHOP-14 or CHOP-21 chemotherapy in European and Australian clinical practice, and UK-specific unit costs, we estimated the total economic impact of FN including care for the initial event as well as downstream FN-related care. The mean economic burden of FN (i.e., the difference in costs between FN patients and comparison patients) was found to be substantial, totaling $£ 5776$ per patient. For FN patients whose index event required hospitalization, economic burden totaled $£ 7259$ (US\$11,610), which is comparable to the estimate (US\$12,397) from the study by Weycker et al. (2008) that employed the same study design and data from a large US healthcare claims database (2001-2003) [9]. Most of the total economic burden was attributable to FN inpatient admissions, since $76 \%$ of index events and $79 \%$ of post-index events required hospitalization, and the (unit) cost of such care is considerably higher than that 
Table 3 Healthcare utilization among FN patients and matched comparison patients

\begin{tabular}{|c|c|c|c|c|c|c|c|c|c|}
\hline \multirow[b]{3}{*}{$\begin{array}{l}\text { Healthcare } \\
\text { Resources }\end{array}$} & \multicolumn{9}{|l|}{ Mean $(95 \% \mathrm{Cl})$} \\
\hline & \multicolumn{3}{|l|}{ Overall* } & \multicolumn{3}{|l|}{ Index Cycle } & \multicolumn{3}{|c|}{ Post-Index Cycle } \\
\hline & $\begin{array}{l}\text { FN Patients } \\
(\mathrm{n}=295)\end{array}$ & $\begin{array}{l}\text { Comparison } \\
\text { Patients } \\
(\mathrm{n}=295)\end{array}$ & Difference & $\begin{array}{l}\text { FN Patients } \\
(\mathrm{n}=295)\end{array}$ & $\begin{array}{l}\text { Comparison } \\
\text { Patients } \\
(\mathrm{n}=295)\end{array}$ & Difference & $\begin{array}{l}\text { FN Patients } \\
(\mathrm{n}=295)\end{array}$ & $\begin{array}{l}\text { Comparison } \\
\text { Patients } \\
(\mathrm{n}=295)\end{array}$ & Difference \\
\hline No. of FN Events & $1.44(1.34,1.56)$ & $0.15(0.10,0.21)$ & $1.29(1.17,1.43)$ & $1.00(1.00,1.00)$ & $0.00(0.00,0.00)$ & $1.00(1.00,1.00)$ & $0.44(0.34,0.56)$ & $0.15(0.10,0.21)$ & $0.29(0.17,0.43)$ \\
\hline $\begin{array}{l}\text { Requiring } \\
\text { Inpatient Care }\end{array}$ & $1.10(1.00,1.21)$ & $0.13(0.08,0.18)$ & $0.98(0.85,1.11)$ & $0.76(0.71,0.80)$ & $0.00(0.00,0.00)$ & $0.75(0.70,0.81)$ & $0.35(0.26,0.45)$ & $0.13(0.08,0.18)$ & $0.22(0.12,0.34)$ \\
\hline $\begin{array}{l}\text { Requiring } \\
\text { Outpatient Care }\end{array}$ & $0.07(0.04,0.11)$ & $0.01(0.00,0.03)$ & $0.06(0.02,0.09)$ & $0.06(0.03,0.09)$ & $0.00(0.00,0.00)$ & $0.06(0.03,0.09)$ & $0.01(0.00,0.03)$ & $0.01(0.00,0.03)$ & $0.00(-0.02,0.02)$ \\
\hline $\begin{array}{l}\text { Requiring } \\
\text { Home Care }\end{array}$ & $0.18(0.12,0.26)$ & $0.01(0.00,0.02)$ & $0.17(0.10,0.24)$ & $0.12(0.08,0.16)$ & $0.00(0.00,0.00)$ & $0.12(0.08,0.16)$ & $0.06(0.02,0.11)$ & $0.01(0.00,0.02)$ & $0.05(0.01,0.10)$ \\
\hline $\begin{array}{l}\text { Requiring Other } \\
\text { Care/No Action }\end{array}$ & $0.05(0.02,0.08)$ & $0.00(0.00,0.00)$ & $0.05(0.02,0.08)$ & $0.04(0.02,0.06)$ & $0.00(0.00,0.00)$ & $0.04(0.02,0.06)$ & $0.01(0.00,0.02)$ & $0.00(0.00,0.00)$ & $0.01(0.00,0.02)$ \\
\hline $\begin{array}{l}\text { Requiring } \\
\text { Unknown Care }\end{array}$ & $0.04(0.02,0.08)$ & $0.00(0.00,0.00)$ & $0.04(0.02,0.08)$ & $0.03(0.01,0.08)$ & $0.00(0.00,0.00)$ & $0.03(0.01,0.05)$ & $0.01(0.00,0.03)$ & $0.00(0.00,0.00)$ & $0.01(0.00,0.03)$ \\
\hline \multicolumn{10}{|l|}{ Use of Healthcare Services } \\
\hline \multicolumn{10}{|l|}{ Inpatient Setting } \\
\hline Admissions & $1.10(1.00,1.21)$ & $0.13(0.08,0.18)$ & $0.98(0.85,1.11)$ & $0.76(0.71,0.80)$ & $0.00(0.00,0.00)$ & $0.75(0.70,0.81)$ & $0.35(0.26,0.45)$ & $0.13(0.08,0.18)$ & $0.22(0.12,0.34)$ \\
\hline Days in Hospital & $6.21(5.28,7.17)$ & $0.63(0.30,1.02)$ & $5.62(4.64,6.62)$ & $4.13(3.56,4.78)$ & $0.00(0.00,0.00)$ & $4.15(3.57,4.81)$ & $2.08(1.40,2.75)$ & $0.63(0.30,1.02)$ & $1.48(0.74,2.23)$ \\
\hline G-CSF (\# admin.) & $0.51(0.31,0.75)$ & $0.05(0.01,0.10)$ & $0.46(0.26,0.69)$ & $0.37(0.22,0.53)$ & $0.00(0.00,0.00)$ & $0.37(0.22,0.53)$ & $0.14(0.03,0.27)$ & $0.05(0.01,0.10)$ & $0.10(-0.03,0.26)$ \\
\hline Filgrastim & $0.33(0.18,0.51)$ & $0.03(0.00,0.08)$ & $0.30(0.15,0.49)$ & $0.23(0.13,0.34)$ & $0.00(0.00,0.00)$ & $0.23(0.13,0.33)$ & $0.10(0.01,0.23)$ & $0.03(0.00,0.08)$ & $0.07(-0.03,0.22)$ \\
\hline Pegfilgrastim & $0.00(0.00,0.01)$ & $0.00(0.00,0.01)$ & $0.00(-0.01,0.01)$ & $0.00(0.00,0.01)$ & $0.00(0.00,0.00)$ & $0.00(0.00,0.01)$ & $0.00(0.00,0.00)$ & $0.00(0.00,0.01)$ & $0.00(-0.01,0.00)$ \\
\hline Other Agent & $0.18(0.06,0.33)$ & $0.01(0.00,0.04)$ & $0.16(0.04,0.31)$ & $0.14(0.04,0.27)$ & $0.00(0.00,0.00)$ & $0.14(0.04,0.25)$ & $0.04(0.00,0.11)$ & $0.01(0.00,0.04)$ & $0.03(-0.03,0.10)$ \\
\hline $\begin{array}{l}\text { IV Antimicrobials } \\
\text { (\# admin.) }\end{array}$ & $4.46(2.68,7.51)$ & $0.42(0.20,0.68)$ & $3.98(2.26,6.89)$ & $2.04(1.64,2.47)$ & $0.00(0.00,0.00)$ & $2.05(1.64,2.48)$ & $2.42(0.83,5.25)$ & $0.42(0.20,0.68)$ & $1.94(0.37,4.70)$ \\
\hline \multicolumn{10}{|l|}{ Outpatient Setting } \\
\hline G-CSF (\# admin.) & $0.27(0.11,0.47)$ & $0.33(0.10,0.62)$ & $-0.06(-0.27,0.13)$ & $0.03(0.00,0.06)$ & $0.00(0.00,0.00)$ & $0.03(0.00,0.06)$ & $0.24(0.08,0.43)$ & $0.33(0.10,0.62)$ & $-0.09(-0.30,0.10)$ \\
\hline Filgrastim & $0.19(0.05,0.37)$ & $0.24(0.02,0.50)$ & $-0.05(-0.25,0.12)$ & $0.02(0.00,0.05)$ & $0.00(0.00,0.00)$ & $0.02(0.00,0.04)$ & $0.17(0.04,0.36)$ & $0.24(0.02,0.50)$ & $-0.07(-0.27,0.11)$ \\
\hline Pegfilgrastim & $0.08(0.03,0.13)$ & $0.09(0.03,0.17)$ & $-0.01(-0.10,0.08)$ & $0.01(0.00,0.03)$ & $0.00(0.00,0.00)$ & $0.01(0.00,0.03)$ & $0.06(0.02,0.12)$ & $0.09(0.03,0.17)$ & $-0.02(-0.11,0.66)$ \\
\hline Other Agent & $0.00(0.00,0.01)$ & $0.00(0.00,0.00)$ & $0.00(0.00,0.01)$ & $0.00(0.00,0.01)$ & $0.00(0.00,0.00)$ & $0.00(0.00,0.01)$ & $0.00(0.00,0.00)$ & $0.00(0.00,0.00)$ & $0.00(0.00,0.00)$ \\
\hline $\begin{array}{l}\text { IV Antimicrobials } \\
\text { (\# admin.) }\end{array}$ & $0.00(0.00,0.00)$ & $0.02(0.00,0.07)$ & $-0.02(-0.07,0.00)$ & $0.00(0.00,0.00)$ & $0.00(0.00,0.00)$ & $0.00(0.00,0.00)$ & $0.00(0.00,0.00)$ & $0.02(0.00,0.07)$ & $-0.02(-0.07,0.00)$ \\
\hline \multicolumn{10}{|l|}{ Home Setting } \\
\hline G-CSF (\# admin.) & $6.29(5.09,7.54)$ & $5.13(4.08,6.28)$ & $1.17(-0.30,2.66)$ & $0.13(0.04,0.22)$ & $0.01(0.00,0.04)$ & $0.11(0.03,0.21)$ & $6.16(4.99,7.41)$ & $5.12(4.07,6.26)$ & $1.06(-0.39,2.52)$ \\
\hline Filgrastim & $2.35(1.60,3.24)$ & $2.20(1.46,3.01)$ & $0.16(-0.89,1.24)$ & $0.07(0.02,0.14)$ & $0.00(0.00,0.03)$ & $0.06(0.00,0.13)$ & $2.28(1.53,3.18)$ & $2.19(1.46,2.99)$ & $0.10(-0.95,1.17)$ \\
\hline Pegfilgrastim & $1.07(0.88,1.29)$ & $0.96(0.77,1.17)$ & $0.12(-0.18,0.38)$ & $0.01(0.00,0.02)$ & $0.00(0.00,0.01)$ & $0.00(-0.01,0.02)$ & $1.07(0.88,1.28)$ & $0.96(0.77,1.17)$ & $0.11(-0.18,0.38)$ \\
\hline
\end{tabular}


Table 3 Healthcare utilization among FN patients and matched comparison patients (Continued)

\begin{tabular}{|c|c|c|c|c|c|c|c|c|c|}
\hline Other Agent & $2.86(1.92,3.97)$ & $1.97(1.19,2.83)$ & $0.90(-0.21,2.03)$ & $0.05(0.00,0.11)$ & $0.00(0.00,0.00)$ & $0.05(0.00,0.11)$ & $2.81(1.89,3.92)$ & $1.97(1.19,2.83)$ & $0.85(-0.25,1.98)$ \\
\hline $\begin{array}{l}\text { IV Antimicrobials } \\
\text { (\# admin.) }\end{array}$ & $1.05(0.68,1.47)$ & $0.03(0.00,0.09)$ & $1.01(0.62,1.45)$ & $0.60(0.37,0.87)$ & $0.00(0.00,0.00)$ & $0.60(0.37,0.86)$ & $0.45(0.17,0.78)$ & $0.03(0.00,0.09)$ & $0.41(0.14,0.74)$ \\
\hline \multicolumn{10}{|l|}{ Other/Unknown Setting } \\
\hline G-CSF (\# admin.) & $0.69(0.41,1.02)$ & $0.87(0.31,1.57)$ & $-0.18(-0.96,0.47)$ & $0.00(0.00,0.01)$ & $0.00(0.00,0.00)$ & $0.00(0.00,0.01)$ & $0.69(0.40,1.02)$ & $0.87(0.31,1.57)$ & $-0.19(-0.97,0.47)$ \\
\hline Filgrastim & $0.45(0.18,0.77)$ & $0.66(0.15,1.33)$ & $-0.23(0.94,0.39)$ & $0.00(0.00,0.01)$ & $0.00(0.00,0.00)$ & $0.00(0.00,0.01)$ & $0.45(0.18,0.76)$ & $0.66(0.15,1.33)$ & $-0.23(0.94,0.39)$ \\
\hline Pegfilgrastim & $0.23(0.14,0.34)$ & $0.15(0.07,0.23)$ & $0.09(-0.04,0.22)$ & $0.00(0.00,0.00)$ & $0.00(0.00,0.00)$ & $0.00(0.00,0.00)$ & $0.23(0.14,0.34)$ & $0.15(0.07,0.23)$ & $0.09(-0.04,0.22)$ \\
\hline Other Agent & $0.01(0.00,0.04)$ & $0.06(0.00,0.17)$ & $-0.04(-0.16,0.03)$ & $0.00(0.00,0.00)$ & $0.00(0.00,0.00)$ & $0.00(0.00,0.00)$ & $0.01(0.00,0.04)$ & $0.06(0.00,0.17)$ & $-0.04(-0.16,0.03)$ \\
\hline $\begin{array}{l}\text { IV Antimicrobials } \\
\text { (\# admin.) }\end{array}$ & $0.00(0.00,0.00)$ & $0.00(0.00,0.00)$ & $0.00(0.00,0.00)$ & $0.00(0.00,0.00)$ & $0.00(0.00,0.00)$ & $0.00(0.00,0.00)$ & $0.00(0.00,0.00)$ & $0.00(0.00,0.00)$ & $0.00(0.00,0.00)$ \\
\hline
\end{tabular}

${ }^{*}$ Mean levels of resource use from index cycle through end of the chemotherapy course. 
Table 4 Healthcare costs among FN patients and matched comparison patients

\begin{tabular}{|c|c|c|c|c|c|c|c|c|c|}
\hline \multirow{3}{*}{$\begin{array}{l}\text { Healthcare } \\
\text { Resources }\end{array}$} & \multicolumn{9}{|c|}{ Mean $(95 \% \mathrm{Cl})$, in British pounds } \\
\hline & \multicolumn{3}{|l|}{ Overall* } & \multicolumn{3}{|l|}{ Index Cycle } & \multicolumn{3}{|c|}{ Post-Index Cycles } \\
\hline & $\begin{array}{l}\text { FN Patients } \\
(n=295)\end{array}$ & $\begin{array}{l}\text { Comparison } \\
\text { Patients } \\
(n=295)\end{array}$ & Difference & $\begin{array}{l}\text { FN Patients } \\
(n=295)\end{array}$ & $\begin{array}{l}\text { Comparison } \\
\text { Patients } \\
(\mathrm{n}=295)\end{array}$ & Difference & $\begin{array}{l}\text { FN Patients } \\
(n=295)\end{array}$ & $\begin{array}{l}\text { Comparison } \\
\text { Patients } \\
(n=295)\end{array}$ & Difference \\
\hline $\begin{array}{l}\text { Total Cost } \\
\text { of FN, Overall }\end{array}$ & $\begin{array}{l}8,066 \\
(7,277,8,882)\end{array}$ & $\begin{array}{l}2,290 \\
(1,923,2,655)\end{array}$ & $\begin{array}{l}5,776 \\
(4,928,6,713)\end{array}$ & $\begin{array}{l}4,051 \\
(3,633,4,485)\end{array}$ & - & $\begin{array}{l}4,051 \\
(3,633,4,485)\end{array}$ & $\begin{array}{l}4,015 \\
(3,374,4,724)\end{array}$ & $\begin{array}{l}2,290 \\
(1,923,2,655)\end{array}$ & $\begin{array}{l}1,725 \\
(978,2,498)\end{array}$ \\
\hline Inpatient Care & $\begin{array}{l}6,007 \\
(5,278,6,771)\end{array}$ & $\begin{array}{l}543 \\
(287,858)\end{array}$ & $\begin{array}{l}5,463 \\
(4,709,6,322)\end{array}$ & $\begin{array}{l}3,942 \\
(3,520,4,372)\end{array}$ & - & $\begin{array}{l}3,942 \\
(3,520,4,372)\end{array}$ & $\begin{array}{l}2,065 \\
(1,478,2,694)\end{array}$ & $\begin{array}{l}543 \\
(287,858)\end{array}$ & $\begin{array}{l}1,522 \\
(900,2,180)\end{array}$ \\
\hline Outpatient Care & $\begin{array}{l}180 \\
(123,252)\end{array}$ & $\begin{array}{l}130 \\
(56,219)\end{array}$ & $\begin{array}{l}50 \\
(-37,144)\end{array}$ & $\begin{array}{l}65 \\
(43,89)\end{array}$ & - & $\begin{array}{l}65 \\
(43,89)\end{array}$ & $\begin{array}{l}115 \\
(62,182)\end{array}$ & $\begin{array}{l}130 \\
(56,219)\end{array}$ & $-15(-97,74)$ \\
\hline Home Care & $\begin{array}{l}1,673 \\
(1,452,1,899)\end{array}$ & $\begin{array}{l}1,447 \\
(1,220,1,674)\end{array}$ & $\begin{array}{l}225 \\
(-78,545)\end{array}$ & $\begin{array}{l}41 \\
(26,59)\end{array}$ & - & $\begin{array}{l}41 \\
(26,59)\end{array}$ & $\begin{array}{l}1,632 \\
(1,409,1,853)\end{array}$ & $\begin{array}{l}1,447 \\
(1,220,1,674)\end{array}$ & $\begin{array}{l}184 \\
(-117,503)\end{array}$ \\
\hline Other/Unknown Care & $\begin{array}{l}207 \\
(135,293)\end{array}$ & $169(97,253)$ & $\begin{array}{l}38 \\
(-67,152)\end{array}$ & $4(2,5)$ & - & $4(2,5)$ & $\begin{array}{l}203 \\
(131,289)\end{array}$ & $\begin{array}{l}169 \\
(97,253)\end{array}$ & $\begin{array}{l}34 \\
(-71,148)\end{array}$ \\
\hline \multicolumn{10}{|c|}{ Total Cost of FN, by Setting of Care } \\
\hline Inpatient Episode & $\begin{array}{l}9,688 \\
(8,709,10,653)\end{array}$ & $\begin{array}{l}2,430 \\
(1,984,2,931)\end{array}$ & $\begin{array}{l}7,259 \\
(6,327,8,205)\end{array}$ & $\begin{array}{l}5,281 \\
(4,810,5,774)\end{array}$ & - & $\begin{array}{l}5,281 \\
(4,810,5,774)\end{array}$ & $\begin{array}{l}4,407 \\
(3,613,5,225)\end{array}$ & $\begin{array}{l}2,430 \\
(1,984,2,931)\end{array}$ & $\begin{array}{l}1,978 \\
(1,262,2,801)\end{array}$ \\
\hline Outpatient Episode & $\begin{array}{l}3,761 \\
(1,908,6,007)\end{array}$ & $\begin{array}{l}1,777 \\
(1,116,2,565)\end{array}$ & $\begin{array}{l}1,984 \\
(-193,4,177)\end{array}$ & $\begin{array}{l}341 \\
(293,435)\end{array}$ & - & $\begin{array}{l}341 \\
(293,435)\end{array}$ & $\begin{array}{l}3,420 \\
(1,529,5,676)\end{array}$ & $\begin{array}{l}1,777 \\
(1,116,2,565)\end{array}$ & $\begin{array}{l}1,644 \\
(-521,3,853)\end{array}$ \\
\hline Home Episode & $\begin{array}{l}3,291 \\
(2,445,4,339)\end{array}$ & $\begin{array}{l}1,826 \\
(993,2,919)\end{array}$ & $\begin{array}{l}1,466 \\
(234,2,495)\end{array}$ & $\begin{array}{l}324 \\
(241,415)\end{array}$ & - & $\begin{array}{l}324 \\
(241,415)\end{array}$ & $\begin{array}{l}2,967 \\
(2,124,4,059)\end{array}$ & $\begin{array}{l}1,826 \\
(993,2,919)\end{array}$ & $\begin{array}{l}1,141 \\
(-99,2,176)\end{array}$ \\
\hline Other/Unknown Episode & $\begin{array}{l}2,211 \\
(1,140,3,425)\end{array}$ & $\begin{array}{l}1,850 \\
(766,3,157)\end{array}$ & $\begin{array}{l}361 \\
(-956,1,650)\end{array}$ & $\begin{array}{l}104 \\
(51,205)\end{array}$ & - & $\begin{array}{l}104 \\
(51,205)\end{array}$ & $\begin{array}{l}2,107 \\
(1,052,3,333)\end{array}$ & $\begin{array}{l}1,850 \\
(766,3,157)\end{array}$ & $\begin{array}{l}258 \\
(-1,031,1,558)\end{array}$ \\
\hline
\end{tabular}


for FN treated in the outpatient or home care settings. As in the study by Weycker and colleagues, while the burden of the initial event was substantial and accounted for the majority of FN-attributable costs (70\%), a significant minority of these costs resulted from follow-on care and subsequent events underscoring the economic importance of accounting for the downstream consequences of this complication. The economic costs of index events averaged $£ 4051$, versus $£ 1725$ for $F N$-related healthcare utilization in post-index cycles.

For several reasons, our estimates of the total economic burden of FN may be conservative. First, for FN events requiring hospitalization, follow-on care that may have occurred in the outpatient setting subsequent to hospital discharge--in the index cycle or subsequently-was not collected during the IMPACT NHL study and thus could not be incorporated into our estimates of disease burden. Second, for all FN events--irrespective of care setting--additional resources that may have been used (e.g., laboratory supplies), and additional services that may have been provided (e.g., drug administration), in the treatment of FN--and that are not included in assumed unit costs/reimbursed values--could not be incorporated due to data limitations. Finally, we erred on the conservative side when making assumptions about the frequency and intensity of resource use outside the hospital setting--where such data were not available from the IMPACT NHL Study--and thus may have underestimated corresponding costs of care. We also note that estimates of disease burden were robust when employing the alternative (i.e., more stringent) matching criteria for FN patients and comparison patients, when admissions designated as "elective" were excluded from estimation of (unit) cost of hospital care, and when using Payment by Results (PbR) tariffs (rather than NHS reference costs) for hospital care (data available in Additional file 1: Tables S6-S8 of the online supplement).

Several limitations of our study are noteworthy. First, although FN patients and comparison patients were matched on several characteristics, it is possible that the two cohorts differed in terms of unobserved characteristics that predispose them to FN. To the extent that FN patients in our study population were more likely to develop FN than comparison patients, some downstream costs--in particular, those occurring in cycles after the one in which the initial (i.e., index) FN event occurred-may not be attributable to the initial FN event per se. Especially problematic in this regard are the costs of subsequent FN events. To what extent does experiencing a first episode of FN increase a patient's risk of subsequent episodes, relative to the extent to which the risk of subsequent episodes is predicted by the same risk factors associated with the initial episode? If largely the latter, the actual burden of chemotherapy-induced FN may be closer to the total costs of the index event plus any additional follow-on care that is directly related to the initial event--most of which, presumably, would occur in the same cycle. Second, it is possible that certain biases in recording may exist such that patients who experience an FN event may be more likely to have FN noted on future encounters versus patients without a history of these complications, all else equal, which could upwardly bias our estimates. Third, although FN-related healthcare utilization appears to be comparable across countries, differences between the UK and other countries in the services or intensity of services that are provided within a given setting (and that are not captured in the study database) are not reflected in study results. Accordingly, caution should be exercised in generalizing the results of this study to other settings. Fourth, we note that FN patients comprise those whose index course was their first course of chemotherapy as well as those who previously received chemotherapy. To the extent that the consequences of FN are different based on prior receipt of chemotherapy, results may not be generalizable to these two subgroups (i.e., subgroups comprising solely patients who previously received chemotherapy and those who did not, respectively). Fifth, we note that various patient- and provider-specific factors may influence the pattern and intensity of FN treatment, on an inpatient and outpatient basis, and that to the extent these factors vary across settings, study results may not be fully generalizable to other patient populations. Sixth, the IMPACT NHL Study was not designed for economic analyses, and thus data on certain types of healthcare utilization (e.g., outpatient visits for follow-on care) were not collected, while other data (e.g., identification and use of IV antimicrobial agents as treatment or prophylaxis) may not always have been collected or classified consistently and comprehensively. Therefore, the results of this analysis may underestimate the total attributable cost of FN. Finally, while our study population comprised adult patients with NHL, we note that the Healthcare Resource Group version 4 (HRG4) code--PA45Z--that was used to cost FN-related inpatient care is grouped in the chapter "Diseases of Childhood and Neonates". We also note, however, that this code has been employed in several single technology appraisals (STAs) and evidence review group's (ERG) reports to cost chemotherapy-related febrile neutropenia among adults with HER2-negative metastatic breast cancer, adults with chronic lymphocytic leukemia, adults with metastatic prostate cancer, and adults with squamous cell carcinoma of the head and neck, respectively [15-18].

\section{Conclusion}

In conclusion, the economic burden of FN among NHL patients in European and Australian clinical practice is 
substantial, and a significant proportion of this burden is due to the downstream consequences of the condition. Studies focusing only on the initial FN event may underestimate the total attributable cost of these complications.

\section{Additional file}

Additional file 1: Table S1. Characteristics of FN and comparison patients matched on basis of original criteria in SSAP; Table S2. UKspecific unit costs of FN-related care; Table S3. Characteristics of FN patients and comparison patients matched on basis of age ( \pm 5 years), tumor stage, chemotherapy regimen, and propensity score; Table S4. Characteristics of full patient population, unmatched patients, and matched patients; Table S5. Healthcare utilization among FN patients and matched comparison patients matched, by country; Table S6. Healthcare costs among FN patients and comparison patients matched on basis of age ( \pm 5 years), country of residence, tumor stage, bone marrow involvement, IPI/FLIPI score, and chemotherapy regimen; Table S7. Healthcare costs among FN patients and matched comparison patients, excluding hospital admissions designated as "elective" from calculation of unit costs; Table S8. Healthcare costs among FN patients and matched comparison patients, using PbR tariffs as basis of unit cost of hospital care.

\section{Authors' contributions}

Authorship was designated based on the guidelines promulgated by the International Committee of Medical Journal Editors (2004). All persons who meet criteria for authorship are listed as authors on the title page. The contribution of each of these individuals to this study--by task--is as follows: conception and supervision (Weycker, Danel), development of design (Weycker, Danel, Marciniak, Bendall), conduct of analyses (Weycker, Lipsitz), interpretation of results (all authors), preparation of manuscript (Weycker, Lipsitz), and review of manuscript (all authors). All authors have read and approved the final version of the manuscript.

\section{Declaration of competing interests}

Derek Weycker and Michael Lipsitz are employed by PAI (Brookline, MA, USA). Aurelie Danel is employed by Amgen Europe (Zug, Switzerland). Anne Marciniak was employed by Amgen Ltd. (Uxbridge, UK) at the time the study was conducted. Kate Bendall is a consultant and funded by Amgen Ltd. (Uxbridge, UK). Ruth Pettengell is employed by St. George's University of London, Department of Haemotology (London, UK), and was a principal investigator for the IMPACT Study.

\section{Declaration of funding}

Funding for this research was provided by Amgen Europe. The study sponsor reviewed and approved the study research plan and study manuscript; data management, processing, and analyses were conducted by PAl, and all final analytic decisions were made by study authors.

\section{Financial support}

Funding for this research was provided by Amgen Europe GmbH to Policy Analysis Inc. (PAI).

\section{Acknowledgements}

None.

\section{Author details}

${ }^{1}$ Policy Analysis Inc. (PAI), 4 Davis Court, Brookline, MA 02445, USA. ${ }^{2}$ Amgen Europe, Dammastrasse 23, Zug 6300, Switzerland. ${ }^{3}$ Amgen Ltd, 1 Uxbridge Business Park, Sanderson Road, Uxbridge UB8 1DH, UK. ${ }^{4}$ Cascade, 3rd Floor, 3 Copthall Avenue, London EC2R 7BH, UK. ${ }^{5}$ Department of Haematology, St. George's University of London, Cranmer Terrace, London SW17 ORE, UK.
Received: 30 January 2012 Accepted: 31 July 2012

Published: 22 August 2012

\section{References}

1. Wildiers H, Reiser M: Relative dose intensity of chemotherapy and its impact on outcomes in patients with early breast cancer or aggressive lymphoma. Crit Rev Oncol Hematol 2011, 77:221-40.

2. Bonadonna G, Moliterni A, Zambetti M, Daidone MG, Pilotti S, Gianni L, Valagussa P: 30 years' follow up of randomised studies of adjuvant CMF in operable breast cancer: cohort study. BMJ 2005, 330:217.

3. Lyman GH, Dale DC, Crawford J: Incidence and predictors of low dose-intensity in adjuvant breast cancer chemotherapy: a nationwide study of community practices. J Clin Oncol 2003, 21:4524-31.

4. Smith TJ, Khatcheressian J, Lyman GH, Ozer H, Armitage JO, Balducci L, Bennett CL, Cantor SB, Crawford J, Cross SJ, Demetri G, Desch CE, Pizzo PA, Schiffer CA, Schwartzberg L, Somerfield MR, Somlo G, Wade JC, Wade JL, Winn RJ, Wozniak AJ, Wolff AC: 2006 update of recommendations for the use of white blood cell growth factors: an evidence-based clinical practice guideline. J Clin Oncol 2006, 24:3187-205.

5. Kwak LW, Halpern J, Olshen RA, Horning SJ: Prognostic significance of actual dose intensity in diffuse large-cell lymphoma: results of a tree-structured survival analysis. J Clin Oncol 1990, 8:963-77.

6. Lyman GH, Kuderer N, Greene J, Balducci L: The economics of febrile neutropenia: Implications for the use of colony-stimulating factors. Eur J Cancer 1998, 34:1857-64.

7. Caggiano V, Weiss RV, Rickert TS, Linde-Zwirble WT: Incidence, cost and mortality of neutropenia hospitalization associated with chemotherapy. Cancer 2005, 103:1916-24.

8. Kuderer NM, Dale DC, Crawford J, Cosler LE, Lyman GH: Mortality, morbidity, and cost associated with febrile neutropenia in adult cancer patients. Cancer 2006, 106:2258-66.

9. Weycker D, Malin J, Edelsberg J, Glass A, Gokhale M, Oster G: Cost of neutropenic complications of chemotherapy. Ann Oncol 2008, 19:454-60.

10. Haioun C, Salar A, Pettengell R: Anemia and erythropoiesis-stimulating agent administration in patients with non-Hodgkin lymphoma treated with cyclophosphamide, doxorubicin, vincristine, and prednisolone \pm rituximab chemotherapy: results from an observational study. Leuk Lymphoma 2011, 52:796-803.

11. Pettengell R, Johnson HE, Lugtenburg PJ, Silvestre AS, Dührsen U, Rossi FG, Schwenkglenks M, Bendall K, Szabo Z, Jaeger U: Impact of febrile neutropenia on R-CHOP chemotherapy delivery and hospitalizations among patients with diffuse large B-cell lymphoma. Support Care Cancer 2012, 20:647-52.

12. Salar A, Haioun C, Rossi FG, Duehrsen U, Pettengell R, Johnsen HE, Jaeger U, Verhoef G, Schwenkglenks M, Bacon P, Bendall K, Lugtenburg $P J:$ The need for improved neutropenia risk assessment in DLBCL patients receiving R-CHOP 21: Findings from clinical practice. Leuk Res 2012. doi:10.1016/j.leukres.2012.02.002.

13. Johnsen HE, Haioun C, Lugtenburg: The importance of G-CSF prophylaxis in R-CHOP-14 therapy for diffuse large B-cell lymphoma in routine clinical practice. Leuk Lymphoma 2012. doi:10.3109/ 10428194.2011.643407

14. Unit Costs of Health and Social Care: Personal Social Services Research Unit (PSSRU):; 2010. Available at: http://www.pssru.ac.uk/pdf/uc/uc2010/uc2010.pdf. Accessed on March 1, 2011.

15. CRD and CHE Technology Assessment Group: Bevacizumab in combination with a taxane for the first-line treatment of HER2-negative metastatic breast cancer: a single technology appraisal:: Centre for Reviews and Dissemination/ Centre for Health Economics; 2010.

16. National Institute for Health and Clinical Excellence, Single Technology Appraisal: Bendamustine for the first-line treatment of chronic lymphocytic leukaemia (Binet stage B or C) in patients for whom fludarabine combination chemotherapy is not appropriate. Available at: http://www.nice.org.uk/ nicemedia/live/12970/52220/52220.pdf. Accessed on July 12, 2012.

17. National Institute for Health and Clinical Excellence, Single Technology Appraisal: Cabazitaxel for the second-line treatment of metastatic hormone refractory prostate cancer. 2011. Available at: http://www.nice. 
org.uk/nicemedia/live/13237/56549/56549.pdf. Accessed on July 12 2012.

18. Greenhalgh J, Bagust A, Boland A, Fleeman N, McLeod C, Dundar Y, Proudlove C, Shaw R: Cetuximab for metastatic and/or recurrent squamous cell carcinoma of the head and neck (SCCHN): A Single Technology Appraisal. The University of Liverpool: LRiG; 2008.

doi:10.1186/1471-2407-12-362

Cite this article as: Weycker et al: Economic costs of chemotherapyinduced febrile neutropenia among patients with non-Hodgkin's

lymphoma in European and Australian clinical practice. BMC Cancer 2012 12:362.

\section{Submit your next manuscript to BioMed Central} and take full advantage of:

- Convenient online submission

- Thorough peer review

- No space constraints or color figure charges

- Immediate publication on acceptance

- Inclusion in PubMed, CAS, Scopus and Google Scholar

- Research which is freely available for redistribution 